Check for updates

Cite this: RSC Adv., 2018, 8, 36879

\title{
Preparation and characterization of soy protein isolate-collagen self-assembled nanomicelles
}

\author{
Ruirui Wang, ${ }^{\text {ab }}$ Hongru Wang, (D) *a Yijun Yao a and Yong Chai ${ }^{a}$
}

This paper describes the preparation and characterization of soy protein isolate (SPI)-collagen selfassembled nanomicelles. Novel SPI-collagen self-assembled nanomicelles via hydrogen bonding and electrostatic interactions were developed. SPI-collagen soluble complexes were formed at $\mathrm{pH} 6.4$, which was lower than the isoelectric point of collagen $(\mathrm{pl}=7.8)$ and higher than that of SPI $(\mathrm{pl}=4.5)$. This research mainly focuses on the intermolecular force between the SPI and collagen. The diameter distribution, critical association concentration, and stability of the SPI-collagen self-assembled nanomicelles were investigated in this research. It was found that the SPI and collagen had hydrogen bonding and electrostatic interactions, and then formed natural complex nanomicelles in a weakly acidic environment. The preparation of SPI-collagen self-assembled nanomicelles was performed in an aqueous environment without surfactants or toxic agents. As a fixing agent, transglutaminase (TGase) promoted the intra- and intermolecular cross-linking of the nanomicelles, which improved the stability of the self-assembled nanomicelles. The SPI-collagen nanomicelles had a good stability. This research may provide a reference for the utilization of SPI-collagen self-assembled nanomicelles.

Received 27th September 2018 Accepted 25th October 2018

DOI: $10.1039 / \mathrm{c} 8 \mathrm{ra0} 0030 \mathrm{e}$

rsc.li/rsc-advances collagen. Novel SPI-collagen nanomicelles self-assembled via hydrogen bonding and electrostatic interactions are developed.

SPI is a mixture of $7 \mathrm{~S}$ ( $\beta$-conglycinin) and $11 \mathrm{~S}$ (glycinin), which are sedimented under different centrifugal forces. SPI has an excellent gelling ability, emulsifying ability, foaming ability and water- and oil-retention. SPI contains all the essential amino acids and can provide a good balance for amino acid compositions. SPI contains physiologically beneficial components that have minimal cholesterin and lessen the risk of hyperlipemia and cardio-cerebro vascular diseases. SPI is an important representative of legume products and has wider application prospects. ${ }^{5}$

As a structural protein, collagen is ubiquitous in skin, bone, connective tissue, and extracellular matrix (ECM). ${ }^{6}$ Collagen is a natural one-dimensional nano-material. ${ }^{7}$ Collagen, which contains three polypeptides, is used as a protein component of biomaterial assemblies. $^{8}$ In vivo, collagen spontaneously assembles into ordered collagen fibers. Then these collagen fibers further form a macro-network structure. It makes tissue have excellent biomechanical properties and structural diversity. In vitro, collagen self-assembled materials are considered as one useful biomaterials because their excellent biocompatibility, biodegradability and weak antigenicity. ${ }^{9}$

Yaks live on "the roof of the world" in rugged environment and are used as basic productive livestock and for the economic livelihood of nomadic pastoralists of the Qinghai-Tibet Plateau. The basic living resources (i.e., milk, meat and hides) of Tibetans living in high-altitude environments are provided by more than 14 million domestic yaks. However, the use of yak
${ }^{a}$ College of Bioresources Chemical and Materials Engineering, Key Laboratory of
Leather Cleaner Production, China National Light Industry, Shaanxi University of
Science \& Technology, Xi'an 710021, Shaanxi, China. E-mail: 21739805@qq.com;
Tel: +8613997280624
${ }^{b}$ College of Chemistry and Chemical Engineering, Qinghai Normal University, Xining
810008, Qinghai, China 
skin is very limited at present and is used as shaving leather, upper leather, leather goods with ethnic characteristics and so on. ${ }^{10}$ Therefore, the development of new biomaterials from yak skin as a raw material will create a new market for the reuse of yak skin.

To the best of our knowledge, the self-assembly interactions of SPI-collagen nanomicelles have not been reported yet. This paper explores the formation of macromolecules via hydrogen bonding and electrostatic interactions of self-assembled polyelectrolyte complexes between SPI and collagen. The objective of this research is to investigate the self-assembly mechanism, diameter distribution, critical association concentration (CAC) and stability of SPI-collagen nanomicelles. The research may provide a reference for the utilization of SPI-collagen nanomicelles.

\section{Experimental section}

\subsection{Materials and chemicals}

SPI was acquired from Shyuanye Biological Science and Technology Co., Ltd. (Shanghai, China). Pepsin (EC 3.4.23.1, $1: 30$ 000) was acquired from Sigma Chemical Co. (St. Louis MO, USA). TGase (EC 2.3.2.13, $100 \mathrm{U} \mathrm{g}^{-1}$ protein) was acquired from Yiming Biological Products Co., Ltd. (Jiangsu, China). Ultrapure water was employed for all solutions except for those used for washing and soaking in which distill water was employed. All the other chemicals reagents used in this research were of analytical reagent grade and were used without further treatment.

\subsection{Extraction of yak skin collagen}

Fresh yak skin was obtained from a trading market in Yushu (Qinghai, China). The excess fat on the yak skin was scraped, and the yak skin was washed in water to remove impurities. Then, the yak skin was dipped in a $0.1 \mathrm{M} \mathrm{NaOH}$ solution with discontinuous stirring to a ratio of $1: 10(\mathrm{w} / \mathrm{v})$ at $20{ }^{\circ} \mathrm{C}$ for $48 \mathrm{~h}$ to get rid of noncollagenous components. The $\mathrm{NaOH}$ solution was replaced per $2 \mathrm{~h}$. The yak skin was cut to a $10 \times 10 \mathrm{~cm}$ block. The degreased skin was rinsed thoroughly until the $\mathrm{pH}$ of the washing solution was 7 .

After pretreatment, the yak skin was dipped in acetic acid $(2 \%, \mathrm{w} / \mathrm{v})$ solution at $10{ }^{\circ} \mathrm{C}$ for $12 \mathrm{~h}$ by discontinuous stirring. The yak skin was incubated with pepsin $\left(20 \mathrm{U} \mathrm{g}^{-1}\right.$ wet skins $)$ at $10{ }^{\circ} \mathrm{C}$ for $18 \mathrm{~h}$ by discontinuous stirring. The extracted collagen solution was filtered by three layers of gauze. The obtained solution was salted out with $\mathrm{NaCl}$. Then precipitates were dialyzed at $10{ }^{\circ} \mathrm{C}$ for $48 \mathrm{~h}$ and then freeze dried. ${ }^{11}$ The molecular weight $\left(M_{\mathrm{w}}\right)$ of the yak skin collagen was $75 \mathrm{kDa}$ and the pI was 7.8 .

\subsection{Preparation of SPI-collagen self-assembled nanomicelles}

The SPI and yak skin collagen solution were prepared separately. The SPI was dispersed in ultrapure water at $70{ }^{\circ} \mathrm{C}$ for $5 \mathrm{~h}$ using magnetic stirring. The yak skin collagen was dissolved in ultrapure water at $40{ }^{\circ} \mathrm{C}$ for $2 \mathrm{~h}$ using magnetic stirring. The final concentrations of the collagen solution and SPI solution were $5 \mathrm{mg} \mathrm{mL}^{-1}$, which could prevent the yak skin collagen and SPI solution forming gel at room temperature. The SPI and collagen solutions were filtrated using Millipore filters $(2.0 \mu \mathrm{m})$ to remove impurities. The SPI and collagen solution were mixed together at a protein ratio of $4: 1$. The SPI-collagen mixtures were prepared by adding the collagen solution to the corresponding amount of the SPI solution. The $\mathrm{pH}$ of the mixtures was adjusted to 6.4. The mixtures were magnetically stirred for $2 \mathrm{~h}$ at $20^{\circ} \mathrm{C}$ and left to rest for at least $24 \mathrm{~h}$ to allow the SPI and collagen to self-assemble completely. Then, the SPI-collagen self-assembled nanomicelles were fixed using TGase for $2 \mathrm{~h}$ at $50{ }^{\circ} \mathrm{C}$. The TGase was deactivated at $80{ }^{\circ} \mathrm{C}$ for $10 \mathrm{~min}$. The selfassembled nanomicelles were freeze-dried and stored at $4{ }^{\circ} \mathrm{C}$.

\subsection{Turbidity measurements}

Transmittance of SPI-collagen self-assembled nanomicelles was measured on a UV-Vis spectrophotometer (Agilent, Cary 5000, USA). Parameter setting: protein concentration: $5 \mathrm{mg} \mathrm{mL} \mathrm{mL}^{-1}$, temperature: $25{ }^{\circ} \mathrm{C}$, wavelength: $600 \mathrm{~nm}$, sample cell: $10 \mathrm{~mm}$ path length quartz cuvette, ultrapure water as reference. Turbidity was indicated $100 \mathrm{~T} \% .^{12}$ Each sample was detected three times and the average value was obtained.

\subsection{Dynamic light scattering (DLS) measurements}

The diameter distribution and polydispersity index (PDI) of the SPI-collagen self-assembled nanomicelles were measured on a Zeta Sizer Nano (Malvern Instrument, ZS 90, UK). Parameter setting: temperature: $25{ }^{\circ} \mathrm{C}$, light source: $4 \mathrm{~mW} \mathrm{He}-\mathrm{Ne}$ laser,

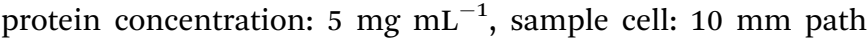
length quartz cuvette, scattering angle: $90^{\circ} .{ }^{13}$ Each sample was detected three times and the average value was obtained.

\subsection{Fluorescence spectroscopy measurements}

Fluorescence spectrum of the SPI-collagen self-assembled nanomicelles was measured on a fluorescence spectrophotometer (Edinburgh, FS 5, UK). Parameter setting: temperature: $25{ }^{\circ} \mathrm{C}$, excitation wavelength: $285 \mathrm{~nm}$, emission wavelength: $338 \mathrm{~nm}$, scanning range: $350-500 \mathrm{~nm}$, scanning rate: 300 $\mathrm{nm} \mathrm{min}^{-1}$, sample cell: $10 \mathrm{~mm}$ path length quartz cuvette. Each sample was detected three times and the average value was obtained. The concentration of the stock solution of pyrene was $2 \times 10^{-5} \mathrm{~g} \mathrm{~mL}^{-1}$, and its final concentration in the SPI-collagen nanomicelles was $2 \times 10^{-7} \mathrm{~g} \mathrm{~mL}^{-1}$. The samples were obtained with various concentrations of the SPI-collagen nanomicelles. The samples that were added to pyrene were homogenized and stored at $4{ }^{\circ} \mathrm{C}$ for $24 \mathrm{~h}$ in total darkness. The fluorescence intensities were recorded at the wavelengths of $373 \mathrm{~nm}\left(I_{1}\right)$, $379 \mathrm{~nm}\left(I_{2}\right), 384 \mathrm{~nm}\left(I_{3}\right), 395 \mathrm{~nm}\left(I_{4}\right)$. All measurements were conducted in triplicate. ${ }^{14}$

\subsection{Fourier transform infrared (FT-IR) spectroscopy measurements}

The FT-IR spectra of the freeze-dried powders of the SPIcollagen self-assembled nanomicelles were measured on 
a spectrometer (Bruker, Vertex 70, Germany). Parameter setting: the film samples: $5 \times 5 \mathrm{~cm}$, scanning range: $400-4000 \mathrm{~cm}^{-1}$, resolution ratio: $4 \mathrm{~cm}^{-1}, \mathrm{KBr}$ tabletting as reference. The freezedried powder was pressed with $\mathrm{KBr}$ to form the pellet, which was used for the FT-IR spectroscopy measurements.

\subsection{Thermogravimetric measurements}

The thermogravimetric analysis of the freeze-dried powders of the SPI-collagen self-assembled nanomicelles was measured on a thermal analyzer instrument (Netzsch, STA 449, Germany). Parameter setting: the samples: $3 \mathrm{mg}$, heating range: $30-700{ }^{\circ} \mathrm{C}$, heating rate: $10^{\circ} \mathrm{C} \mathrm{min}^{-1}$, nitrogen flow rate: $20 \mathrm{~mL} \mathrm{~min}^{-1}$, empty crucible as reference.

\section{Results and discussion}

\subsection{Turbidity of the nanomicelles}

Because the $M_{\mathrm{w}}$ of extracted collagen in our study is close to that of SPI, collagen and SPI can form polyelectrolyte complexes by a self-assembly method. The turbidity is frequently used to study polyelectrolyte complexes. The collagen and SPI formed a polyelectrolyte complex by a self-assembly method in a precise $\mathrm{pH}$ range ( $\mathrm{pH}$ 5.1-7.28). The turbidity of the complexes at different $\mathrm{pH}$ values is shown in Fig. 1. Critical $\mathrm{pH}$ values (the formation of soluble $\left(\mathrm{pH}_{\mathrm{c}}\right)$ and insoluble $\left(\mathrm{pH}_{\varphi}\right)$ complexes) were shown the intersection point of two turbidity curve tangents by graphic method. ${ }^{1}$ According to Fig. 1, the interaction between the SPI and collagen in a solution underwent three stages with the variation of the $\mathrm{pH}$ of the solution. In the first stage, there was no interaction between the SPI and collagen. In the second stage, the SPI and collagen formed complexes by a self-assembly method. In the third stage, the SPI and collagen underwent a phase separation of proteins. The SPI and collagen formed soluble complexes between pH 6.2 and 7.28 by a self-assembly method. As the $\mathrm{pH}$ continued to decrease, the SPI and collagen formed insoluble complexes between pH 5.1 and 6.2. There were hydrogen bonding and electrostatic attraction between the SPI and collagen to form the complexes. When the $\mathrm{pH}$ was under a boundary value $\left(\mathrm{pH}_{\mathrm{b}}\right.$ 5.1), the molecular interactions between homologous protein molecules became stronger and led to a phase separation. The results indicate that SPI-collagen self-assembled nanomicelles were formed at $\mathrm{pH}$ $6.2-7.28$.

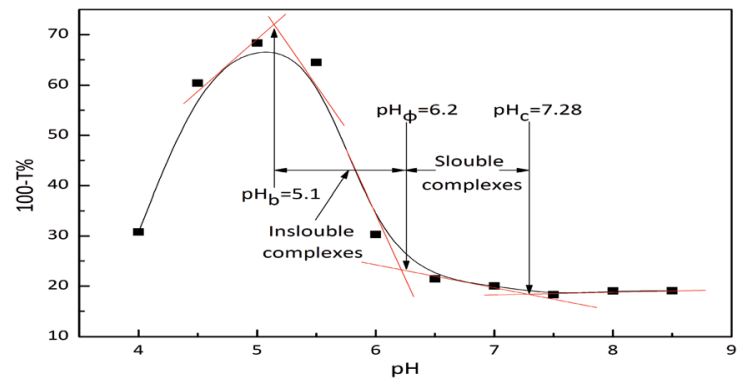

Fig. 1 Turbidity plots at different $\mathrm{pH}$ values with a collagen-SPI mixing ratio of 0.25 .

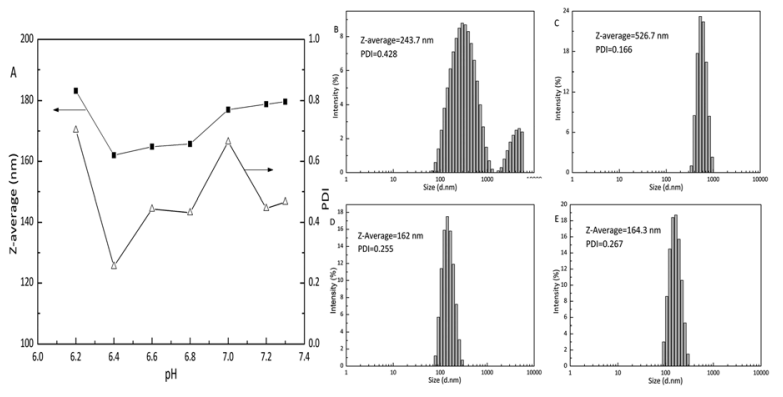

Fig. 2 Diameter and PDI plots of the nanomicelles (A), the diameter distribution of the SPI (B), collagen (C), unfixed nanomicelles (D) and TGase-fixed nanomicelles (E).

\subsection{Diameter distribution of the nanomicelles}

The diameter and PDI of the SPI-collagen self-assembled nanomicelles at $\mathrm{pH}$ 6.2-7.28 are shown in Fig. 2(A). The diameter of the SPI-collagen nanomicelles reached the minimum at $\mathrm{pH}$ 6.4. The results illustrate that the charge density of the oppositely charged SPI and collagen was close to equilibrium at pH 6.4. The two kinds of protein molecules had the strongest hydrogen bonding and electrostatic interactions and formed the minimum particle size and PDI at pH 6.4. Therefore, $\mathrm{pH}$ 6.4 was selected as the optimum condition for preparing SPIcollagen nanomicelles, at which $\mathrm{pH}$ the formation of SPIcollagen soluble complexes was further investigated.

The diameter distribution of the SPI, collagen, unfixed SPIcollagen self-assembled nanomicelles and TGase-fixed nanomicelles at $\mathrm{pH} 6.4$ are shown in Fig. 2(B-E). The average diameter of the SPI-collagen nanomicelles was smaller than that of the SPI and collagen, indicating that the SPI and collagen had strong hydrogen bonding and electrostatic interactions in the self-assembly process. Between the active groups (carbonyl, amino and hydroxyl groups) of the SPI and of the collagen could produce hydrogen bonding interaction. At the same time, the partial negative charge of the carboxyl groups in the SPI molecules and the partial positive charge of the amino groups in the collagen molecules could produce electrostatic interaction. The collagen and SPI formed macromolecules via hydrogen bonding and electrostatic interactions in the weakly acidic environment. The average diameter of unfixed self-assembled nanomicelles is $162 \mathrm{~nm}$, and the average diameter of the TGase-fixed selfassembled nanomicelles is $164.3 \mathrm{~nm}$. The nanomicelles had a high intensity and a narrow PDI, indicating that the collagen and SPI could form stable nanomicelles by the self-assembly method in a weakly acidic environment. The results indicate that this self-assembly was performed under mild conditions without the addition of surfactants.

\subsection{Critical association concentration (CAC) of the nanomicelles}

The CAC is an important parameter in the investigation of the aggregation behavior of biological macromolecules. ${ }^{\mathbf{1 4}}$ Pyrene was used as a fluorescent probe for the formation of a hydrophobic microenvironment in the SPI-collagen self-assembled nanomicelles. The pyrene transferred from an aqueous phase 


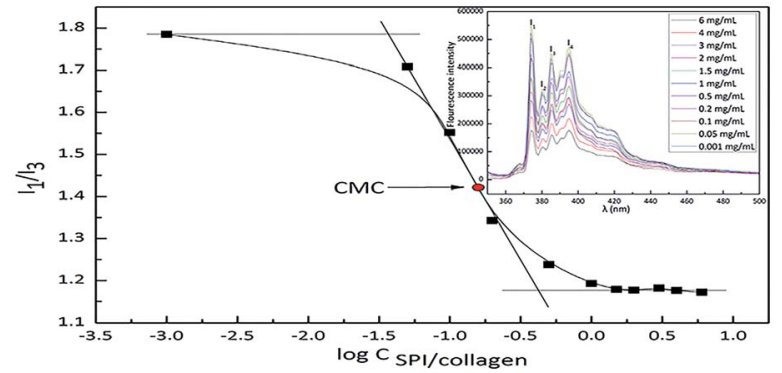

Fig. 3 Fluorescent emission spectra and plots of the pyrene fluorescence $I_{1} / l_{3}$ ratio versus the concentration of the nanomicelles.

to a hydrophobic microenvironment, accompanied by significant changes of the fluorescence intensity. The intensity ratio of the first vibronic peaks to the third vibronic peaks $\left(I_{1} / I_{3}\right)$ can be used to evaluate the pyrene microenvironment's polarity. ${ }^{15}$ The emission spectra of the pyrene and the plots of the pyrene fluorescence $I_{1} / I_{3}$ ratio versus the concentration of SPI-collagen self-assembled nanomicelles are shown in Fig. 3; there are four peaks at 373, 380, 384 and $395 \mathrm{~nm}$. This result indicates that pyrene exhibited a stable fluorescent property in the SPIcollagen complex system. The pyrene fluorescence intensity ratio $\left(I_{1} / I_{3}\right)$ continuously decreased from 1.785 to 1.172 with the increase in the concentration of SPI-collagen self-assembled nanomicelles. This response suggests that the polarity of the solution decreased as the pyrene molecules entered the hydrophobic microenvironment of the SPI-collagen self-assembled nanomicelles. As shown into Fig. 3, the abrupt midpoint of the curve was $0.18 \mathrm{mg} \mathrm{mL}^{-1}$, suggesting that the CAC value of the SPI-collagen self-assembled nanomicelles might be $0.18 \mathrm{mg}$ $\mathrm{mL}^{-1}$.

\subsection{Self-assembly mechanism of the nanomicelles}

By analyzing the turbidity, diameter and CAC of the SPI-collagen self-assembled nanomicelles, we further propose a formation mechanism of SPI-collagen nanomicelles (Fig. 4). The selfassembly of SPI and collagen in an aqueous solution can be explained by their molecular structures. The SPI and collagen molecules showed a random coil structure in the aqueous solution ${ }^{14}$ because the regular structure of the SPI and collagen were destroyed after extraction and heating. The numerous amino and carboxylic groups locating in the amino acid units afford plentiful binding sites. ${ }^{16}$ In mixing the collagen and SPI, the $\mathrm{pH}$ of the obtained soluble complexes was in the range of 6.2-7.28. In this range, the $\mathrm{pH}$ was lower than the $\mathrm{pI}$ of the collagen ( $\mathrm{pI}_{\mathrm{yak} \text { skin collagen }}=7.8$ ) and higher than the $\mathrm{pI}$ of the SPI $\left(\mathrm{pI}_{\mathrm{SPI}}=4.5\right)$; the collagen peptides with partial positive electrostatic charge and the SPI peptides with partial negative electrostatic charge formed macromolecules via electrostatic interactions. But because the deprotonation of the carboxyl group of the SPI and the protonation of the amino group of the collagen were not strong in the range of 6.2-7.28, it could be deduced that hydrogen bonding interaction was major force during formation of the complexes (Fig. 4). The collagen peptides competed with the SPI peptides and bound to the interior sites of the SPI micelles during formation. The peptides

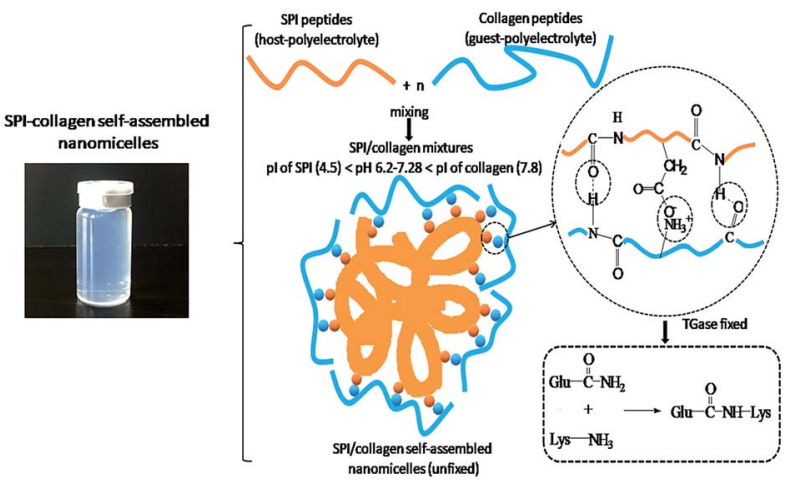

Fig. 4 Diagram of the mechanism for formation of the nanomicelles.

were bonded and in situ assembled by hydrogen bonding interactions of the active groups (carbonyl, amino and hydroxyl groups) of the SPI and of the collagen and electrostatic interactions of the partial amino groups of the collagen and the carboxylic groups of the SPI. Then, all the negative charges in the SPI were neutralized by the positive charges in the collagen. These hydrogen bonding and electrostatic interactions promoted the formation of stable SPI-collagen nanomicelles. In these complexes, the SPI played the role of host-polyelectrolyte, and collagen played the role of guest-polyelectrolyte. The nanomicelles were formed consequently through hydrogen bonding of the active groups and electrostatic interactions of opposite charges in the aqueous medium. The strong hydrogen bonding interactions played important roles in the formation of the SPI-collagen self-assembled nanomicelles. ${ }^{3}$

However, the application of self-assembled nanomicelles has been severely limited due to them being easily deformed or destroyed when they are removed from the preparation conditions. Therefore, various fixing strategies have been proposed to obtain a stable material. However, normal fixing agents, such as glutaraldehyde, were proven to be cytotoxic. To reduce the toxicity, TGase as a new fixing agent was used in this research. After adding TGase, the TGase could catalyze an acyl-transfer reaction between the $\gamma$-carboxamide group of peptide-bound glutamine residues and the $\varepsilon$-amino group of the lysine residue in the nanomicelles to form inter- or intramolecular $\varepsilon-$ $\left(\gamma\right.$-glutamyl) lysine isopeptide bond. ${ }^{17}$ The inter- or intramolecular crosslinking promoted the stability of the selfassembled nanomicelles.

\section{5. $\quad$ FT-IR spectrum of the nanomicelles}

The FT-IR spectra of the collagen, SPI, unfixed SPI-collagen selfassembled nanomicelles and TGase-fixed SPI-collagen selfassembled nanomicelles are shown in Fig. 5. The spectrum of the collagen had five most characteristic bands. The amide A band of the collagen at $3275 \mathrm{~cm}^{-1}$ is ascribed to the stretching vibrations of $\mathrm{N}-\mathrm{H}$ groups. The amide $\mathrm{B}$ band of the collagen at $2940 \mathrm{~cm}^{-1}$ is ascribed to the asymmetrical stretching of $\mathrm{CH}_{2}{ }^{-}$. The amide I band of the collagen at $1633 \mathrm{~cm}^{-1}$ is ascribed to the stretching of $\mathrm{C}=\mathrm{O}$. The amide II band of the collagen at $1535 \mathrm{~cm}^{-1}$ is ascribed to the bending of $\mathrm{N}-\mathrm{H}$ and the stretching 

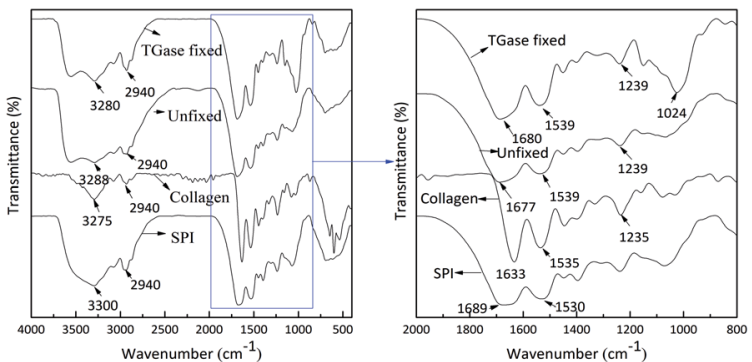

Fig. 5 FT-IR spectra of the collagen, SPI, unfixed nanomicelles and TGase fixed nanomicelles.

of $\mathrm{C}-\mathrm{N}$. The amide III band of the collagen at $1235 \mathrm{~cm}^{-1}$ is ascribed to the $\beta$-sheet of the protein. ${ }^{18}$

A free $\mathrm{N}-\mathrm{H}$ stretching vibration occurred in the range $3400-$ $3440 \mathrm{~cm}^{-1}$. If the $\mathrm{N}-\mathrm{H}$ group on a peptide was participated in a hydrogen bond, its peak was shown a red-shift. The amide A bands of the self-assembled nanomicelles were at 3288 and $3280 \mathrm{~cm}^{-1}$. This observation indicates that more $\mathrm{N}-\mathrm{H}$ groups of the SPI and collagen were participated in forming hydrogen bond. The presence of hydrogen bonding leads to a wider absorption peak. The amide I bands of the self-assembled nanomicelles were at 1677 and $1680 \mathrm{~cm}^{-1}$. The nanomicelles had a lower wavenumber for the amide I bands, suggesting an increased interactions of $\mathrm{C}=\mathrm{O}$ with the adjacent groups by hydrogen bonds and electrostatic interactions between the $\mathrm{C}=\mathrm{O}$ of the SPI and active groups of the collagen. The slight increase in the intensity of the amide I bands and the amide II bands that are associated with the amino and carboxyl groups revealed that the collagen conformational changes are induced by the presence of the SPI. This result indicated that the interactions are mainly due to hydrogen bonding interactions of the active groups (carbonyl, amino and hydroxyl groups) of the SPI and of the collagen and electrostatic interactions of the carboxylic groups of the SPI and the partial amino groups of the collagen (the interactions are shown in Fig. 4).

The TGase fixed nanomicelles showed a significant increase in the intensity of the amide I bands. This finding suggested that the TGase caused cross-linking between the SPI and collagen. The cross-linking might change the nanomicelle conformation. The increase in the intensity of the amide I bands and amide III of the TGase fixed nanomicelles indicated that the $\beta$-sheet content increased in the secondary structure. The additional absorption peaks of the self-assembled nanomicelles fixed with TGase at approximately $1024 \mathrm{~cm}^{-1}$ are associated with $\mathrm{C}-\mathrm{O}$ stretching and $\mathrm{C}-\mathrm{O}$ bending, ${ }^{19}$ suggesting that the interactions between the hydroxyl groups of the SPI and collagen occurred via hydrogen bonding or the saccharide structure of the carbohydrates that are incorporated into the commercial TGase. ${ }^{13}$

The above results indicated that the collagen and SPI could form soluble complexes at $\mathrm{pH} 6.4$ by a self-assembly method. The SPI-collagen nanomicelles are soluble aggregates whose assembly was driven by noncovalent forces (such as hydrogen bonding and electrostatic interactions).

\subsection{Thermal performance of the nanomicelles}

The thermogravimetry (TG) curves of the SPI-collagen selfassembled nanomicelles are shown in Fig. 6(A). The weight loss of all samples underwent three stages when it was heated from $30{ }^{\circ} \mathrm{C}$ to $700{ }^{\circ} \mathrm{C}$. The weight loss of the samples was less than $10 \%$ in the first stage $\left(<240{ }^{\circ} \mathrm{C}\right)$. This result was due to the evaporation process of the bound moisture. The weight loss of the samples rapidly increased in the second stage $\left(240-400{ }^{\circ} \mathrm{C}\right)$. The weight loss of the samples was more than $40 \%$. This step was the process of water molecule removal by combining neighboring hydroxyl groups in collagen and SPI molecules. The weight loss of the samples tended to slow down and eventually stabilized in the third stage $\left(>400{ }^{\circ} \mathrm{C}\right)$. The weight loss of the samples was less than $13 \%$. The weight loss of the SPI-collagen nanomicelles was markedly reduced, indicating that the collagen significantly increased the thermal stability of the SPI at pH 6.4.

From Fig. 6(B), the derivative thermo gravimetry (DTG) curve of the unfixed SPI-collagen nanomicelles had a small peak temperature of decomposition at $107^{\circ} \mathrm{C}$ and a high peak temperature of decomposition at $316{ }^{\circ} \mathrm{C}$. This result shows that the nanomicelles began to decompose when the temperature approached $107{ }^{\circ} \mathrm{C}$. The neighboring hydroxyl groups combined to generate water molecules that were removed, which occurred by exothermic reactions. The decomposition rate reached the maximum at $316^{\circ} \mathrm{C}$ and the equilibrium at $450^{\circ} \mathrm{C}$. The DTG curves of the TGase fixed SPIcollagen nanomicelles had a small peak temperature of decomposition at $136{ }^{\circ} \mathrm{C}$ and a high peak temperature of decomposition at $316^{\circ} \mathrm{C}$. However, the DTG curves of the SPI had a small peak temperature of decomposition at $85^{\circ} \mathrm{C}$ and a high peak temperature of decomposition at $308^{\circ} \mathrm{C}$, which manifested that the thermal stability of the SPI-collagen selfassembled nanomicelles was higher than that of the SPI. The TGase further improved the thermal stability of the selfassembled nanomicelles, suggesting that the collagen and SPI formed soluble aggregates by noncovalent forces (such as hydrogen bonding and electrostatic interactions). Then, the collagen and SPI were cross-linked by the fixation with TGase. The soluble aggregates had a good thermal stability. The SPIcollagen self-assembled nanomicelles could withstand the heat processes during processing and application.
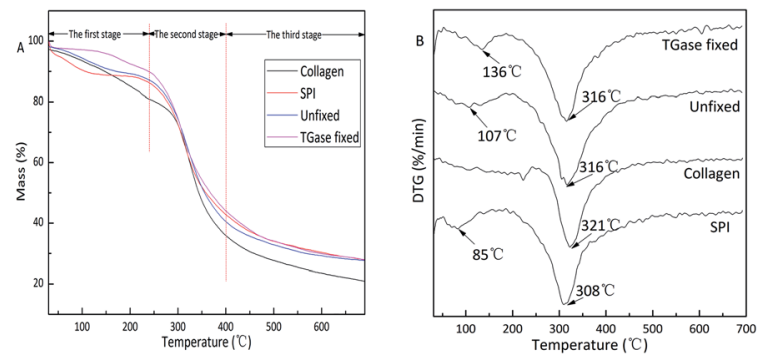

Fig. 6 TG and DTG curves of the collagen, SPI, unfixed nanomicelles and TGase fixed nanomicelles. 

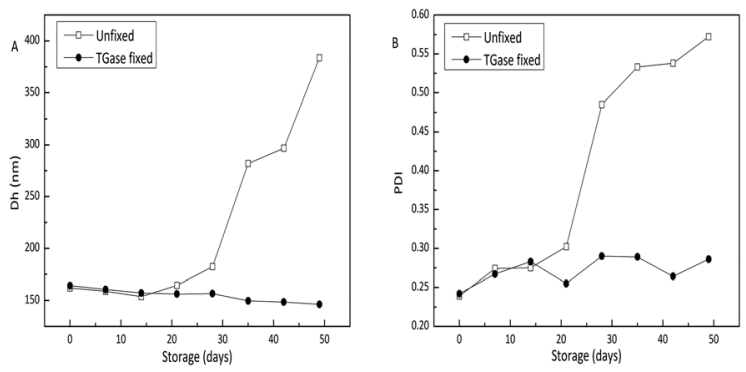

Fig. 7 Plots of the diameter (A) and PDI (B) of the nanomicelles during 50 days of preservation.

\subsection{Storage stability of the nanomicelles}

The self-assembled nanomicelles might usually be deformed or destroyed when they are removed from the preparation environment. The stabilization of nanomicelles has been a major problem. In this study, TGase was used to fix SPIcollagen nanomicelles by enzyme catalyzed crosslinking. The storage stability of SPI-collagen nanomicelles is shown in Fig. 7. The diameter and PDI of the unfixed nanomicelles after 50 days were approximately 2 times greater than that of the freshly prepared samples. However, the diameter and PDI of the TGase-fixed nanomicelles after 50 days were essentially unchanged. These results show that the TGase-fixed nanomicelles had better storage stability than the unfixed nanomicelles. The SPI emulsion had a significantly improved stability in the presence of collagen due to the collagen and SPI forming soluble, self-assembled complexes in the weakly acidic environment. TGase, as a fixing agent, effectively improved the storage stability of the SPI-collagen nanomicelles (Fig. 7). Therefore, the results suggest that the SPIcollagen nanomicelles have good storage stability and can be used in weakly acidic conditions.

\subsection{Thermal stability and freeze-dried stability of the nanomicelles}

The thermal stability $\left(100^{\circ} \mathrm{C}\right)$ and freeze-dried $\left(-20^{\circ} \mathrm{C}\right)$ stability of the SPI-collagen self-assembled nanomicelles are shown in Fig. 8. The diameter of the SPI-collagen unfixed nanomicelles increased after the heat treatment and freeze-drying treatment. However, the diameters of the TGase-fixed SPI-collagen

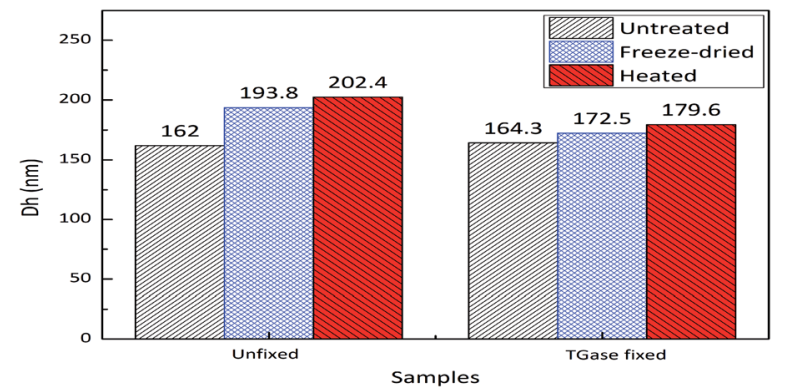

Fig. 8 Diameters of the nanomicelles were recorded after $10 \mathrm{~min}$ at $100{ }^{\circ} \mathrm{C}$ or after $24 \mathrm{~h}$ at $-20^{\circ} \mathrm{C}$. nanomicelles were essentially unchanged. These results indicated that the collagen played an important role of chaperone during the heating or cooling treatments of the SPI-collagen self-assembled nanomicelles. During heating or cooling, more positive charged groups on the collagen and more negative charged groups on the SPI were exposed and electro statically interacted. Finally, the soluble aggregates were formed by noncovalent interactions. TGase, as a fixing agent of selfassembled nanomicelles, exhibited a superior fixing effect. The SPI-collagen nanomicelles had a good thermal stability and freeze-drying stability.

\section{Conclusions}

Novel soy protein isolate-collagen nanomicelles self-assembled via hydrogen bonding and electrostatic interactions were developed. The nanomicelles were prepared with a collagen-soy protein isolate mixing ratio of 0.25 at $\mathrm{pH} 6.4$ with a protein concentration of $5 \mathrm{mg} \mathrm{mL} \mathrm{m}^{-1}$. Turbidity tests and dynamic light scattering tests demonstrated that the active groups (carbonyl, amino and hydroxyl groups) of the soy protein isolate peptide chains and of the collagen peptide chains exhibited strong hydrogen bonding interactions, and the partial carboxyl groups on the surface of the soy protein isolate peptide chains exhibited electrostatic interactions with the partial amino groups on the collagen peptide chains and then formed natural polymer nanomicelles at $\mathrm{pH}$ 6.4. The changes in the Fourier transform infrared spectra further confirmed the interactions. The fluorescence spectra tests measured the critical association concentration of the soy protein isolate-collagen nanomicelles to be $0.18 \mathrm{mg} \mathrm{mL}^{-1}$. As a fixing agent, TGase promoted intraand intermolecular cross-linking of nanomicelles, which improved the stability of the soy protein isolate-collagen nanomicelles. The soy protein isolate-collagen nanomicelles had good thermal and storage stabilities. The preparation of nanomicelles was performed in an aqueous environment without surfactants or toxic agents. Future work will investigate the encapsulation and delivery properties of these soy protein isolate-collagen nanomicelles.

\section{Conflicts of interest}

There are no competing interests to report.

\section{Acknowledgements}

Qinghai Natural Science Foundation (2016-ZJ-949Q, 2019-ZJ7071) and Qinghai Normal University Research Foundation (2018-ZR-008) financially supported our work.

\section{References}

1 N. G. Voron'ko, S. R. Derkach, Y. A. Kuchina and N. I. Sokolan, Carbohydr. Polym., 2016, 138, 265-272.

2 S. R. Derkacha, N. G. Voron'ko, Y. A. Kuchina, D. S. Kolotova, A. M. Gordeeva, D. A. Faizullin, Y. A. Gusev, Y. F. Zuev and O. N. Makshakova, Carbohydr. Polym., 2018, 197, 66-74. 
3 P. R. Sarika and N. R. James, Carbohydr. Polym., 2016, 148, 354-361.

4 T. B. Goudoulas and N. Germann, Food Hydrocolloids, 2017, 66, 49-60.

5 Y. Yuan, Z. L. Wan, X. Q. Yang and S. W. Yin, Food Res. Int., 2014, 55, 207-214.

6 M. Fang, L. G. Elizabeth, K. M. Eryn, O. G. Bradford and H. M. Banaszak, Langmuir, 2013, 7, 2330-2338.

7 T. Y. Kim, I. Sridharan, B. Zhu, J. Orgel and R. Wang, Mater. Sci. Eng., C, 2015, 49, 281-289.

8 C. Y. Liu, H. Y. Liu, A. Xu, K. Y. Tang, Y. Huang and C. Lu, J. Alloys Compd., 2017, 714, 522-529.

9 H. C. Goo, Y. S. Hwang, Y. R. Choi, H. N. Cho and H. Suh, Biomaterials, 2003, 24, 5099-5113.

10 G. Wiener, Food Sci., 2011, 343-350.

11 M. Q. Xu, L. X. Wei, Y. C Xiao, H. T. Bi, H. X. Yang and Y. Z. Du, Food Hydrocolloids, 2018, 75, 13-21.
12 C. Qi, Y. Chen, J. H. Huang, Q. Z. Jin and X. G. Wang, J. Sci. Food Agric., 2012, 92, 787-793.

13 X. Z. Su, H. R. Wang and M. Huang, J. Nanomater., 2014, 1-8. 14 Y. Q. Wang, C. Ye, L. H. Wu and Y. Z. Hu, J. Pharm. Biomed. Anal., 2010, 53, 235-242.

15 G. B. Ray, I. Chakraborty and S. P. Moulik, J. Colloid Interface Sci., 2006, 294, 248-254.

16 H. R. Du, K. F. Huang, W. Dong and B. Y. Geng, Electrochim. Acta, 2018, 279, 66-73.

17 J. Calvarro, T. P. Palacios and J. Ruiz, Int. J. Gastron. Food. Sci., 2016, 5, 27-32.

18 F. R. Xing, S. F. Zhang, J. Z. Li, L. Li and J. Y. Shi, Polym. Compos., 2018, 39, 942-949.

19 W. Y. Weng and H. B. Zheng, Food Chem., 2015, 169, 255260. 